\title{
Reuse of Cardiac Rhythm Control Devices: A Review AKMM Islam, ${ }^{1}$ M Ullah, ${ }^{2}$ US Khan, ${ }^{3}$ AAS Majumder ${ }^{2}$ \\ ${ }^{1}$ Department of Cardiology, Jessore Medical College, ${ }^{2}$ Department of Cardiology, NICVD, \\ ${ }^{3}$ Department of Cardiology, Delta Medical College.
}

\begin{abstract}
:
Keywords:

Cardiac

Cardiac rhythm control devices i.e. pacemakers, implantable cardioverter-defibrillators (ICDs) and biventricular pacemakers are at times lifesaving treatment and prevention of arrhythmia. But, despite definite indications, many people in the developing countries cannot afford these devices due to high cost, arrhythmia, Pacemaker, Implantable CardioverterDefibrillators, Recycling and succumb to premature death. On the other hand, after implantation, pacemakers and ICDs are sometimes underutilized because of premature explantation due to death of the recipient, device infection, or upgradation, leaving a considerable extent of serviceable battery life unused. Majority of these explanted devices are simply thrown away. Though made for single use, a growing body of evidence indicates the safety, efficacy and feasibility of reimplantation of the prematurely explanted devices in patient in need who is otherwise unable to afford a new one. Patients, physicians, morticians, and even the general public support the idea of device donation and recycling. A number of organizations around the world have engaged themselves in this benevolent effort, and $t$

he feasibility of device reutilization programme has already been established. However, some logistic, legal and ethical concerns are yet to be solved. The statutory bodies and professional organizations should address the issue of device recycling to solve the unsolved issues and formulate standard practice guidelines. In that case, such medical wastes may turn into invaluable resources, and help ensuring equitable medical care throughout the world.
\end{abstract}

(Cardiovasc. j. 2013; 6(1): 52-59)

\section{Introduction:}

Arrhythmia is a common cardiac condition. Besides pharmacotherapy, devices are often required for management. Permanent pacemakers are used for bradyarrhythmias and sick-sinus syndrome. Internal cardioverterdefibrillators (ICDs) are often life-saving in malignant tachyarrhythmias. Biventricular pacemakers are indicated in heart failure with dyssynchrony for cardiac resynchronization therapy (CRT).

Despite well-established indications, many patients in low- and middle-income countries cannot afford these devices, due to their high cost. ${ }^{1}$ On the other hand, for various reasons, these cardiac rhythm control devices are sometimes explanted prematurely, with considerable extent of their battery life remaining. As a result, potential scopes are created for their reuse in patients unable to afford a new device. However, reuse of a device made for single-use is associated with considerable ethical and legal barriers.

\section{Epidemiology}

Each year approximately 1 million individuals worldwide die due to a lack of access to pacemakers. ${ }^{2,3}$ There are no data on the number of patients dying from sudden cardiac death due to lack of ICD therapy. ${ }^{4}$ The majority of these preventable deaths occur in developing countries. According to the 11th World Survey of Cardiac Pacing and Implantable CardioverterDefibrillators: Calendar Year 2009-A World Society of Arrhythmia's Project there is marked disparity in rate of utilization of these often lifesaving devices between the developed and the developing countries around the world. Germany had the highest new pacemaker implants per million population (927) followed by France (782), the USA (767), and Italy (744). PPM implantation 
rate per million population in developing countries is very low e.g. Bangladesh (5), India (17), Pakistan (4), Indonesia (2), Mayanmar (2), Sudan (5), Peru (30). Large ICD implanters per million population included USA (434), Germany (290), the Netherlands (220), and Italy (174). Again ICD implantation rate per million population was Bangladesh (1), India (1), Pakistan $(<1)$, Indonesia (<1), Mayanmar $(<1)$, Sudan $(<1)$, Peru (1). This disparity is explained in part by cost. Cardiac rhythm control devices usually cost hundreds or thousands of Dollars, which is far beyond the means of a large proportion of the population living in these countries.

\section{Basics of Cardiac Rhythm Control Devices}

Modern rhythm control devices are operated by lithium-iodine batteries. On an average, a modern pacemaker works for 7-10 years, ${ }^{6,7}$ and the median longevity of an AICD is less than 5 years to 7.6 years, ${ }^{8-10}$ depending on the rate of use. In comparison to the longevity of the pacemakers, that of ICDs is less predictable because of high individual variability of use. At the end of battery life, they need explantation and implantation of a new device, often the generator, if indication persists. But, before reaching this end of life, many devices are explanted prematurely, mainly due to death of the recipient, device infection, or device upgradation.

In an older retrospective study of 169 consecutive pacemaker patient deaths, $58 \%$ mortality was within 2 years of the last generator implant; $19 \%$ within 6 months and $38 \%$ within 1 year. ${ }^{11} \mathrm{In}$ another study, average time between implantation and explantation of 69 pacemakers was 46 months i.e. $<4$ years ( 1 day to 11 years) and at the time of explantation, all pacemakers had adequate battery reserve and functioning programmes. ${ }^{6}$ Similar observations have been reported in case of ICDs. Out of 106 explanted ICDs, the mean time the device was in use before that first patient died was 522 days, i.e. $<2$ years. ${ }^{12}$ In another recent study involving 3176 rhythm control devices donated for the reutilization program, approximately $21 \%$ of donated devices and $30 \%$ of donated biventricular ICDs possessed an adequate battery life for potential reuse. ${ }^{1}$ So, current cardiac rhythm control devices often have substantial remaining serviceable battery life after the death of the patient. ${ }^{12}$ And this creates the scope of recycling these devices. Actually, in a series of 453 reused pacemakers with 17 years follow-up in India, the average battery life of refurbished device was 8.3 years. ${ }^{13}$ Device shelf life is estimated at between 12 and 18 months, after which a device is considered expired due to loss of battery capacity and inability to ensure sterility. ${ }^{14}$ Manufacturers and hospitals routinely donate late model and expired pulse generators and leads to charity organizations for use overseas.

Disposal of Cardiac Rhythm Control Devices After the end of battery life, the old generator is replaced by a new one electively. In case of premature death of the recipient, disposal of the devices is predominantly determined by the local customs, religious factors, and emotion of the near-relatives. In Muslim and also in Catholic world, dead bodies are usually buried, but in some countries like India, dead bodies are predominantly cremated. In case of burial, pacemakers and ICDs are usually buried with the dead-body, only some are removed on family's request. Burial of the device may be detrimental to the environment, as the heavy metals of the device have the potential to leech into burial soil and also water supplies. ${ }^{15}$ For cremation, they are taken out of the body in order to avoid explosion. ${ }^{16}$ In a recent survey involving 90 funeral directors in Michigan, 114 patients with devices and 1,009 members of the general population, cremation rate was $35 \%$, the majority of devices (84\%) were discarded as medical waste or stored with no intended purpose, $89 \%$ of funeral directors, $87 \%$ of device patients and $71 \%$ of the general population expressed a desire to donate devices to the needy patients. ${ }^{17}$

In developed countries, disposal of the dead bodies by cremation appears to be increasing; the estimated cremation rate for 2025 is $59 \% .{ }^{18}$ Therefore, nearly 2 million individuals with pacemakers and ICDs are expected to be cremated in 2025, and their device will have to be explanted as per routine protocol. ${ }^{1}$ So, only explantation after death in developed countries is, and will continue to be a huge source of rhythm control device for recycling. 
For pacemaker infection, the standard practice is to explant the device not to be reused, and after control of infection, a new device is implanted. Rates of device infections requiring explantation were $1.82 / 1000$ after the first implantation and 5.32/1000 after replacement in a series of 46299 pacemakers implanted between 1982 and 2007 in Denmark. ${ }^{19}$

The mortality rate associated with device infection was $8.2 \%, 4.6 \%$ and $5.8 \%$ for pacemaker, ICD and defibrillating biventricular pacemaker (CRT-D) respectively, in another cohort. ${ }^{20}$ In a separate study, the mean time between implantation and explantation for device infection was 52 days. ${ }^{21}$ So, logically, some of these explanted devices should have adequate residual battery life for reuse, and after resterilization, may be a potential source of recycled devices. Sometimes, the pacemakers are replaced by biventricular pacemakers or ICDs. In case of such upgradation, the older device may have adequate battery life, making it potentially reusable.

The ownership of the rhythm control devices and responsibilities for their proper disposal vary among the countries. Low and middle income countries mostly do not have laws regulating single-use devices and handling standard for medical devices. ${ }^{22}$ Even in the United States, no federal law establishes postmortem property rights pertaining to explanted medical devices. ${ }^{22}$ On the other hand, ownership is ascribed to the implanting centre in Sweden, ${ }^{23,24}$ or to the patient in United Kingdom, ${ }^{25}$ the Netherlands and Canada. ${ }^{26}$ Sometimes the explanted device is needed for medicolegal purpose.

The idea of pacemaker reuse appears to be well accepted by the general population. ${ }^{27}$ According to a survey of the general population in 2009, $71 \%$ of the general public and $87 \%$ of patients with pacemakers are willing to donate a device to people in need in another country, if given the chance. ${ }^{28}$

\section{Concerns of Reuse of Cardiac Rhythm Control Devices}

Cardiac rhythm control devices are manufactured for single-use only. There remains a doubt whether these devices can be adequately resterilized for subsequent use. Body fluid and proteins may remain specially at the ports of attachment with the leads, which cannot be well reached by the disinfectant. ${ }^{4}$ So, the risk of transmission of prion disease or CreutzfeldtJakob disease remains, at least theoretically. Pacemakers and ICDs have got a definite battery life of their own, and depending on the length and frequency of use, depletion of variable portion of energy occurs. Further depletion occurs during the transitional period between explantation and reimplantation. So, generators with partially depleted energy reserve may not serve for a reasonable period of time in the subsequent recipient in case of device recycling. Another concern is risk of device malfunctioning. In case of first use, each rhythm control device, specially the ICD and biventricular pacemaker, is programmed according to the individual need. After explantation, when the device is reimplanted, it may be unsuitable for optimal reprogramming as per the need of the second recipient, and the risk of inappropriate shock is considerable. However, these devices can be used as simple pacemakers switching off the extra functions. ${ }^{22,28}$ Cardiac rhythm control devices are in the way of continuous improvement of design and performance. Older devices are interrogated to find out the pitfalls and solutions. Practice of recycling may hinder to returning of the explanted device to the manufacturers, thereby chance quality improvement may be lost. That is why the Heart Rhythm Society (HRS) officially recommends return of all explanted devices to the manufacturers. ${ }^{29}$ Manufactures could theoretically donate returned pulse generators after extensive testing, but some of the tests destroy device functionality. ${ }^{22}$ Insurance coverage is provided for the brand new devices; lack of insurance coverage may be a problem, specially in those countries where this is available. Manufacturer's warranty is provided for new devices only; recycled device lacks any warranty, and this may be a problem for the consumers of these sorts of sophisticated and expensive devices. Use of an explanted device with potential health risks in a human being may raise ethical issues, as well. In case of complications or device malfunctions, such an 
'unethical' practice may lead to take legal action by the recipient, as well as, by the manufacturers against the implanting physicians, the institutions and the people concerned with device recycling. Also, explantation to reimplantation of rhythm control device is a team work, involving a chain of donors, physicians, charity organizations, healthcare institutions and the recipients. Coordination and maintenance of transparency may be difficult; there is chance of faults in surveillance of this huge task.

\section{Scientific Evidence behind Reuse of Cardiac Rhythm Control Devices}

The reuse of donated rhythm control devices in low and middle income countries has been practiced for many years. ${ }^{22}$ Reuse of pacemakers was started in Scandinavian countries probably in late sixties. In Sweden pacemakers have been reused for decades. The favourable provisions of Swedish law probably contributed to the tradition to reuse of pacemakers there. By Swedish law, all implanted pacemakers and defibrillators ought to be explanted after the death of the recipient to avoid device explosion during cremation. Explantation of a pacemaker was not defined as an autopsy and can be performed by any physician or technician in a pathology department. Moreover, not the patient but the implanting centre was the owner of the implanted pacemaker. When Sweden became a member of the European Common Market in 1994, the Medical Board of Health elaborated rules for the use of medical-technical products in concurrence with those in the Common Market. As a result, the reuse of pacemakers dropped from $14 \%$ of all primary implantations and replacements in 1991 to 8\% in $1994-5 .{ }^{30}$

Pacemaker reimplantation was found to be safe and feasible without excess infection or device malfunction in different countries around the world. ${ }^{27}, 30-34$ Some studies reported no statistical difference in survival between device reuse and new implantation. ${ }^{35,36}$ Two metaanalyses have also demonstrated the safety and efficacy of pacemaker reuse. ${ }^{37,38}$ The metaanalysis of 18 studies $^{38}(\mathrm{n}=2270)$ involving pacemaker reuse found infection $1.97 \%$, device malfunction $0.68 \%$; no significant difference in infection rate between pacemaker reuse and new device implantation (odds ratio, 1.31 [0.50 to 3.40], $\mathrm{p}=0.580$ ). However, compared with new device implantation, there was an increased risk for malfunction in the reuse group (odds ratio, 5.80 [1.93 to 17.47 ], $\mathrm{p}=0.002$ ), mainly driven by abnormalities in set screws and nonspecific device "technical errors." In a recently published study from Mexico, a cohort of 603 consecutive patients (study group $n=307$ received resterilized pacemakers, and the control group $(n=296)$ received a new pacemaker) from 2000 to 2010 was studied to see the combined end point of 3 major outcomes i.e. unexpected battery depletion, infection, and device dysfunction. Other than the expected shorter battery life, reuse of pacemaker generators was not inferior to the use of new devices. ${ }^{39}$ Data regarding safety and efficacy of reuse of ICDs and biventricular pacemakers are less robust. In a single-center, retrospective study in India involving 81 indigent persons with mean follow-up of 824.9 days, the reused ICDs functioned normally and delivered life-saving therapies, without an increased risk for complications. ${ }^{12}$

\section{Device Selection for Reuse of Cardiac Rhythm Control Device}

No uniform criteria defining suitability for reimplantation of explanted cardiac rhythm control devices exist. Criteria for reuse of pacemakers in the Karolinska Hospital, Sweden included: no signs of pacemaker malfunction in previous recipient, no signs of damage to visible parts of pacemaker, normal parameters on control measurements with pacemaker system analyser, estimated running time $>1 / 3$ of total life-expectancy of pacemaker, or pacemaker implanted $<3$ years in previous recipient. ${ }^{30}$ Kantharia et al. ${ }^{40}$ used explanted pacemakers with battery life $>3$ years whereas Hasan et al. ${ }^{27}$ used presence of $>70 \%$ of battery life remaining for reimplantation. Adequate battery life was defined as $>75 \%$ or $>4$ years of estimated longevity in a more recent reutilization programme involving 3176 devices (65\% pacemakers, $21 \%$ ICDs, $12 \%$ biventricular ICDs, and $3 \%$ biventricular pacemakers). ${ }^{1}$ For reuse of ICDs, presence of battery voltage of at least $3.0 \mathrm{~V}$ or projected longevity of at least 3 years 
was a prerequisite of selection of device in a recent study from India. ${ }^{12}$ Clearly, presence of standard, uniform criteria would make the practice of device recycling more efficient and confident.

\section{Resterilization Protocol}

No uniform protocol for resterilization of explanted cardiac rhythm control devices exists. As a result, different institutions use different methods of sterilization. In Sweden, for reuse, explanted pacemaker was programmed to nominal values and put in a solution containing phenoxypropanol and benzalconium chloride for 12 hours, then rinsed under running water and dried. It was subsequently wiped with $70 \%$ ethanol, air-dried, and finally packed, labelled and sterilized in ethylene oxide. ${ }^{30} \mathrm{~A}$ more recent report described the resterilization procedure for ICDs. ${ }^{12}$ After explantation, the ICDs were cleaned with saline and sealed them in biohazard plastic bags. They were then interrogated, patient and lead information were deleted, reprogrammed to non-pacing mode, and sensing, alarm and shocking functions were turned off. Before reimplantation, the ICDs were immersed in hydrogen peroxide for 10 minutes, in povidoneiodine for 2 minutes, and then rinsed with running water for 2 minutes and air-dried. Finally, the device was sterilized with ethylene oxide gas at $38^{\circ} \mathrm{C}$ for 4 hours followed by 6 hours of aeration. Demonstration of efficacy of the resterilization procedure, whatever it is, is crucial for avoiding post-reimplantation infection. Cultures are done in this regard. ${ }^{27}$ Clearly, presence of a standard resterilization protocol would make the practice of device recycling more confident.

\section{Current Status of Reuse of Cardiac Rhythm Control Devices}

Some charitable organizations and physicians are actively involved in recycling the cardiac rhythm control devices around the globe. ${ }^{41}$ They collect the explanted pacemakers and ICDs from the persons, hospitals, funeral homes and sometimes from the manufacturers, analyze them, and finally send them for reuse in medically underserved nations. In the United States, World Medical Relief and Heartbeat International are 2 such organizations. World
Medical Relief collects and distributes recycled medical and dental equipment, medical supplies, laboratory instruments, and medicines to third world and developing nations around the world. ${ }^{42}$ Project My Heart Your Heart is a joint collaboration between citizens, physicians, and funeral directors in the state of Michigan, the University of Michigan Cardiovascular Center, World Medical Relief, and teaching hospitals in the Philippines, Vietnam, Ghana, and Nicaragua. The purpose of the project is to create a central organization that obtains pacemakers postmortem for evaluation and subsequent sterilization while creating a distribution network for safe reuse. ${ }^{43}$ Heartbeat International receives the donation of new pacemakers and ICDs (often close to expiration from a sterility standpoint) from the manufacturers, and distributes the devices to the global network of heart centers via local Rotary chapters. ${ }^{2}$ Over the years, it has donated approximately 11,000 devices in more than 25 developing countries. Another charitable organization Heart Too Heart, collects used pacemakers from funeral homes and family members of the deceased (or new ones that for a variety of reasons cannot be used in the United States) since 1994. Those that can be reused are sent to Eastern Europe and South America, where they are implanted for free in poor patients. In 13 years, it has sent between 1,400 and 1,600 pacemakers overseas. ${ }^{44}$

Physicians' attitude appears to be in favour of device recycling for helping the poor nations. In a recent survey, the HRS physician-members responded positively to the charitable overseas donation of explanted, sterilized, usable pacemakers and ICDs. Out of the 429 responding HRS members, $9.6 \%$ had previously implanted a recovered, resterilized pacemaker. ${ }^{45}$

The 1985 North American Society of Pacing and Electrophysiology Policy Conference concluded that reuse is not a risk factor for device infection. ${ }^{46}$ Subsequently, the 2002 American College of Cardiology/American Heart Association/North American Society of Pacing and Electrophysiology guideline update for implantation of cardiac pacemakers acknowledged that pacemaker reuse "may 
eventually add significantly to the costeffectiveness of cardiac pacing." ${ }^{47}$ However, ACC/AHA/HRS 2008 Guidelines for DeviceBased Therapy of Cardiac Rhythm Abnormalities $^{48}$ and 2012 ACCF/AHA/HRS Focused Update of the 2008 Guidelines for Device-Based Therapy of Cardiac Rhythm Abnormalities ${ }^{49}$ have not mentioned the issue of reuse of cardiac rhythm control devices.

At present, reuse of cardiac rhythm control devices is not sanctioned by any governing or regulatory body in the USA or European Union. Pacemakers and ICDs are currently labelled as single-use devices, ${ }^{50}$ and the Unite States Food and Drug Administration (USFDA) specifically prohibits reuse, referring to it as "an objectionable practice" ${ }^{11}$ However, in the USA, there are no federal prohibitions on harvesting used pacemakers for implantation abroad.

\section{Bangladesh Perspective}

Bangladesh is a developing country. Despite recent socio-economic advancement, rhythm control device implantation rate is still very low: 5 and 1 per million population for pacemaker and ICD respectively. ${ }^{5}$ National Institute of Cardiovascular Diseases (NICVD) is the main centre of the country which provides device therapy for arrhythmia. Besides this, an increasing number of Government and private institutions are rendering such treatment. Data regarding reuse of pacemakers and ICDs are lacking. However, device recycling occurs in a small scale for long. No standard practice for explantation and re-implantation exists to date. No rules and regulations dictate the ownership and disposal of the devices. Patients are presumably thought to be the owner of the device, and the family members determine the fate of it, in case of 'premature' death of the recipient. Emotion of the next of kin and social, as well as, religious factors probably play important role in final disposition of the device post-mortem. The device is usually buried with the dead body. Cremation is an infrequent way of disposal of the body after death. Unlike the developed world, infection is presumably the main source of reusable explanted device. After control of infection the resterilized device is sometimes reimplanted into the same recipient, specially in case of financial stringency. Sometimes, with informed consent, the explanted device is reimplanted in a patient who is otherwise unable to afford such a device. There is a limited Government supply of pacemakers, almost free of cost, for the very poor people. Right now, no regular collaboration exists between any Bangladeshi organization and device-donating charitable organizations like World Medical Relief or Heartbeat International. For resterilization at NICVD, explanted device is at first kept in Chlorhexidine Gluconate-cetrimide solution (Savlon) for 2 days, then cleaned by syringing with water, followed by sterilization in ethylene oxide gas for 24 hours. The device is then kept in air for 24 hours, and then again sterilized by ethylene oxide gas for 3 hours. ${ }^{52}$

\section{Future Directions}

Though not recognized by regulatory bodies, reuse of cardiac rhythm control device is a reality, and appears to be scientifically sound and economically viable practice specially in developing countries. However, considerable obstacles remain on the way of more widespread use of recycled device. More research, specially large, multicentre clinical trials should further evaluate its safety and efficacy. Statutory bodies should deal with this issue, and formulate standard practice guidelines. The recipients of the pacemakers and ICDs may be offered a "living will for devices" for proper disposal of the rhythm control device. Donating patients, retrieving physicians, funeral homes and the device manufacturers should, by law, both national and international, be considered free of liability ${ }^{12}$ Side by side, only responsible physicians, institutions and organizations with adequate knowledge, motivation and logistic support should be allowed for device recycling. Like cornea banking, pacemaker and ICD banks can be established for collection and storage of devices obtained from local and international resources. Proper surveillance should be maintained to prevent possible commercialization of the recycled devices. Legislation concerning device donation and reuse will remove many hurdles. More systematic method of transportation recognized by Customs would 
help decrease the shelf time and may lead to greater device longevity in the second recipient. ${ }^{12}$ Like organ donation, device donation can be a subject of social movement throughout the world, which will help removing social stigma and add fuel to the practice of device recycling.

\section{Conclusion:}

Reuse of cardiac rhythm control device is no more a 'hide and seek' game. It is not an uncommon practice in many parts of the world. It has got enormous potential to save numerous lives specially in underdeveloped nations, facilitating equity of medical care. However, concerns remain regarding safety and efficacy of this practice, many aspects of it is still uncertain, or poorly defined. Constructive criticism of the existing practice, and further research are needed to deal with the residual concerns. In that case, device recycling will certainly, open up new avenue in medical philanthropy in future.

\section{Conflict of Interest - None.}

\section{References:}

1. Baman TS, Crawford T, Sovitch P, et al. Feasibility of postmortem device acquisition for potential reuse in underserved nations. Heart Rhythm 2012 Feb;9(2): 211-214.

2. Heartbeat International. Heartbeat-our mission. Available at: http://www.heartbeatintl.org/mission.htm. Accessed Jul 13, 2013.

3. Mond HG, Mick W, Maniscalco BS. Heartbeat International: making "poor" hearts beat better. Heart Rhythm 2009;6:1538-1540.

4. Van Heuverswyn FE, Timmers L, Stroobandt RX, et al. Implantable cardioverter-defibrillators: is there life after death? Pacing Clin Electrophysiol 2013 Jan;36(1):2-6.

5. Mond HG, Proclemer A. The 11th world survey of cardiac pacing and implantable cardioverter-defibrillators: calendar year 2009 - a World Society of Arrhythmia's project. Pacing Clin Electrophysiol 2011 Aug;34(8): 1013-1027.

6. Suvarna SK, Start RD, Tayler DI. A prospective audit of pacemaker function, implant lifetime, and cause of death in the patient. J Clin Pathol 1999;52:677-680.

7. Hauser RG, Hayes DL, Kallinen LM, et al. Clinical experience with pacemaker pulse generators and transvenous leads: an 8-year prospective multicenter study. Heart Rhythm 2007 Feb;4(2):154-160.

8. Thijssen J, Borleffs JW, van Rees JB. Implantable cardioverter defibrillator longevity under clinical circumstances: an analysis according to device type, generation, and manufacturer. Heart Rhythm 2012;9: 513-519.

9. Schaer BA, Koller MT, Sticherling C, et al. Longevity of implantable cardioverter-defibrillators, influencing factors, and comparison to industry-projected longevity. Heart Rhythm 2009;6:1737-1743.
10. Horlbeck FW, Mellert F, Kreuz J, et al. Real-world data on the lifespan of implantable cardioverter-defibrillators depending on manufacturers and the amount of ventricular pacing. J Cardiovasc Electrophysiol 2012;23:1336-1342.

11. Pringle RA, Leman RB, Kratz JM, et al. An argument for pacemaker reuse: pacemaker mortality in 169 patients over ten years. Pacing Clin Electrophysiol 1986 Nov;9(6 Pt 2):1295-1298.

12. Pravi BB, Lokhandwala Y, Kulkami GV, et al. Reuse of explanted, resterilized implantable cardioverter defibrillators. Ann Intern Med 2012; 157:542-548.

13. Balachander J, Anilkumar R, Sethuraman KR, et al. Efficacy and safety of refurbished pacemakers: 17 years follow-up of an international collaborative programme. Stimucoeur 2003;31:190-193.

14. Senaratne J, Irwin ME, Senaratne MPJ. Pacemaker longevity: are we getting what we are promised? Pacing Clin Electrophysiol 2006;29:1044-1054.

15. Bramstedt KA. Harms and responsibilities associated with battery-operated implants (BOI): who controls postmortem explantation? Pacing Clin Electrophysiol 2013 Jan; 36(1):7-10.

16. Gale CP, Mulley GP. Pacemaker explosions in crematoria: problems and possible solutions. J R Soc Med 2002;95: 353-355.

17. Gakenheimer L, Lange DC, Romero J, et al. Societal views of pacemaker reutilization for those with untreated symptomatic bradycardia in underserved nations. J Interv Card Electrophysiol 2011 Apr;30(3):261-266.

18. Cremation Association of North America. Available at: http:/ /www.cremationassociation.org/. Accessed Jul 13, 2013.

19. Johansen JB, Jørgensen OD, Møller M, et al. Infection after pacemaker implantation: infection rates and risk factors associated with infection in a population-based cohort study of 46299 consecutive patients. Eur Heart $J$ 2011 Apr;32(8):991-998.

20. Sohail MR, Henrikson CA, Braid-Forbes MJ, et al. Mortality and cost associated with cardiovascular implantable electronic device infections. Arch Intern Med 2011 Nov 14;171(20):1821-1828.

21. Klug D, Balde M, Pavin D, et al. PEOPLE Study Group. Risk factors related to infections of implanted pacemakers and cardioverter-defibrillators: results of a large prospective study. Circulation 2007;116:1349-1355.

22. Kirkpatrick JN, Papini C, Baman TS, et al. Reuse of pacemakers and defibrillators in developing countries: logistical, legal, and ethical barriers and solutions. Heart Rhythm 2010 Nov;7(11):1623-1627.

23. Ryden L. Re-use of devices in cardiology. Eur Heart $J$ 1998; 19:1628-1631.

24. Government Bill 1994/95:148 s. 67-68: Transplants and autopsies, etc. [I lagens f " orarbeten (prop. 1994/95:148 s. 67-68): Transplantationer och obduktioner m.m.].

25. Medicines and Healthcare products Regulatory Agency. Department of Health and Social Security Health Notice HN(83)6. Available at: http://www.mhra.gov.uk/home/ 
groups/dts-bi/documents/websiteresources/con123256.pdf. Accessed Jul 13, 2013.

26. Jackson M. Issues affecting refurbishment and re-use of pacemakers. Australian Health Rev 1996;19:68-80.

27. Hasan R, Ghanbari H, Feldman D, et al. Safety, efficacy, and performance of implanted recycled cardiac rhythm management (CRM) devices in underprivileged patients. Pacing Clin Electrophysiol 2011 Jun;34(6):653-658.

28. Baman TS, Romero A, Kirkpatrick JN, et al. Safety and efficacy of pacemaker reuse in underdeveloped nations: A case series. J Am Coll Cardiol 2009;54:1557-1558.

29. Carlson MD, Wilkoff BL, Maisel WH, et al. American College of Cardiology Foundation; American Heart Association; International Coalition of Pacing and Electrophysiology Organizations. Recommendations from the Heart Rhythm Society Task Force on Device Performance Policies and guidelines endorsed by the American College of Cardiology Foundation (ACCF) and the American Heart Association (AHA) and the International Coalition of Pacing and Electrophysiology Organizations (COPE). Heart Rhythm 2006;3:1250-1273.

30. Linde CL, Bocray A, Jonsson H, et al. Re-used pacemakers-as safe as new? A retrospective case-control study. Eur Heart J 1998 Jan;19(1):154-157.

31. Havia T, Schuller H. The re-use of previously implanted pacemakers. Scand J Thorac Cardiovasc Surg Suppl 1978;22:33-34.

32. Kruse IM. Experiences from the reuse of implantable pulse generators. Clin Prog 1985;3:61-63.

33. Pescariu S, Stiubel M, Cozma D, et al. La réutilisation des pacemakers, une alternative pour les personnes a 'ge'es de'munies: etude re'trospective. Stimucouer 2003;31: 186-189.

34. Rosengarten M, Chiu R, Hoffman R. A prospective trial of new versus refurbished cardiac pacemakers: a Canadian experience. Can J Cardiol 1989;5:155-160.

35. Mugica J, Duconge R, Henry L. Survival and mortality in 3,701 pacemaker patients: arguments in favor of pacemaker reuse. Pacing Clin Electrophysiol 1986;9: 1282-1287.

36. Panja M, Sarkar CN, Kumar S, et al. Reuse of pacemaker. Indian Heart J 1996;48:677-680.

37. Romero J, Meier P, Gakenheimer L, et al. Pacemaker reutilization is a safe and effective means of delivering electrophysiological healthcare in Third World Countries a meta-analysis. Circ Cardiovasc Qual Outcomes 2011;4:AP96.

38. Baman TS, Meier P, Romero J, et al. Safety of pacemaker reuse: a meta-analysis with implications for underserved nations. Circ Arrhythm Electrophysiol 2011 Jun;4(3): 318-323.

39. Nava S, Morales JL, Márquez MF, et al. Reuse of pacemakers: comparison of short and long-term performance. Circulation 2013 Mar 19;127(11):1177-1183.

40. Kantharia BK, Patel SS, Kulkarni G, et al. Reuse of explanted permanent pacemakers donated by funeral homes. Am J Cardiol 2012 Jan 15;109(2):238-240.

41. Stanyon R. Donation of explanted pacemakers for reuse in underserved nations. J Healthc Risk Manag 2010;29(3):4,6-8,14.
42. World Medical Relief, Inc. Available at: http:// www.worldmedicalrelief.com. Accessed Jul 13, 2013.

43. Project My Heart Your Heart. Michigan Cardiovascular Outcomes Research and Reporting Programs. Available at: http://mcorrp.org/welcome/?q=node/40. Accessed Jul 13, 2013.

44. Mitka M. Death not necessarily end for heart devices. JAMA 2007; 297:144-145.

45. Crawford TC, Desai N, Hagan L, et al. Heart Rhythm Society members' views on pacemaker and implantable cardioverter defibrillator reutilization. Heart Rhythm Society 2013 Scientific Sessions; May 8, 2013; Denver, CO. Abstract PO01-48. Available at: http://conference-cast.com/ HRS/common/presentation-detail.aspx/8/23/1066/6746. Accessed Jul 13, 2013.

46. Boal BH, Escher DJ, Furman S, et al. Report of the policy conference on pacemaker re-use sponsored by the North American Society of Pacing and Electrophysiology. Pacing Clin Electrophysiol 1985 Mar;8(2):161-163.

47. Gregoratos G, Abrams J, Epstein AE, et al. ACC/AHA/ NASPE 2002 guideline update for implantation of cardiac pacemakers and antiarrhythmia devices: summary article: a report of the American College of Cardiology/American Heart Association Task Force on Practice Guidelines (ACC/AHA/NASPE Committee to Update the 1998 Pacemaker Guidelines). J Am Coll Cardiol 2002;40: 1703-1719.

48. Epstein AE, Dimarco JP, Ellenbogen KA, et al. American College of Cardiology; American Heart Association Task Force on Practice Guidelines; American Association for Thoracic Surgery; Society of Thoracic Surgeons. ACC/ AHA/HRS 2008 Guidelines for device-based therapy of cardiac rhythm abnormalities. Heart Rhythm 2008 Jun;5(6):e1-62.

49. Epstein AE, DiMarco JP, Ellenbogen KA, et al. American College of Cardiology Foundation; American Heart Association Task Force on Practice Guidelines; Heart Rhythm Society. 2012 ACCF/AHA/HRS focused update incorporated into the ACCF/AHA/HRS 2008 guidelines for device-based therapy of cardiac rhythm abnormalities: a report of the American College of Cardiology Foundation/ American Heart Association Task Force on Practice Guidelines and the Heart Rhythm Society. J Am Coll Cardiol 2013 Jan 22;61(3):e6-75.

50. U.S. Food and Drug Administration. CPG Sec. 300.500. Reprocessing of Single Use Devices. Silver Spring, MD: U.S. Food and Drug Administration;2010. Available at: www.fda.gov/ I C E C I/ ComplianceManuals/ CompliancePolicyGuidanceManual/ucm073887.htm. Accessed July 13, 2013.

51. U.S. Food and Drug Administration. CPG Sec. 310.100. Pacemaker Reuse. Silver Spring, MD: U.S. Food and Drug Administration; 2009. Available at: www.fda.gov/ICECI/ ComplianceManuals/CompliancePolicyGuidanceManual/ ucm073891.htm. Accessed Jul 13, 2013.

52. Khatun N. Sister-in-Charge of cath lab, Natonal Institute of Cardiovascular Diseases, Dhaka, Bangladesh. Personal communication. Jul 14, 2013. 